\title{
Rasgos propuestos para una maestría en cuidado de la vida como necesidad del contexto*
}

\author{
Marina Sánchez Sanabria \\ Francia Esther Herrera Salazar ${ }^{2}$ \\ Mariemma Socarrás Vega ${ }^{3}$
}

Recibido: 10-06-2020

Aceptado: 05-07-2020

\section{Resumen}

Compete a la universidad - por responsabilidad social frente a un contexto afectado por deficientes comportamientos socioeconómicos, débil capacidad productiva y competitiva, y con problemas sociales que reflejan su multifactorialidad en el cuidado de la salud- liderar la creación de una maestría multidisciplinar iniciando con un estudio de viabilidad que responda a desafíos globales como diálogo intercultural, salud pública, cambio climático, seguridad alimentaria, gestión de recursos naturales, entre otros, que promueva el desarrollo del pensamiento crítico, creativo e innovador con responsabilidad ciudadana, ético, y fomente la cultura ciudadana. Como parte de esta investigación, se analizan las necesidades de formación y tendencias educativas que permitan

\footnotetext{
* Artículo avance de resultados del estudio "Viabilidad para la creación de un programa de maestría en la Facultad de Ciencias de la Salud, Universidad Popular del Cesar", aprobada por la Facultad de Ciencias de la Salud y la Oficina de Arcadia de la Universidad Popular del Cesar.
}

1. Enfermera, UIS. Magíster en Salud Pública, Universidad del Valle; magíster en Educación con Énfasis en Cognición, Uninorte. Doctora en Ciencias de la Educación, URBE. Docente titular, carrera de Énfermería, Unicesar. Investigadora sénior, grupo Control de Calidad de los Procesos en Salud (categoría A Colciencias).

Correo electrónico: marinasanchez@unicesar.edu.co

ORCID: http://orcid.org/0000-0002-8148-4478

2. Enfermera, Unicesar. Magíster en Enfermería Materno-Infantil, Universidad del Valle; magíster en Educación con Enfasis en Cognición, Uninorte. Doctora en Ciencias de la Educación. Docente titular de Unicesar. Investigadora asociada. Directora del grupo de investigación Control de Calidad de los Procesos en Salud.

Correo electrónico: franciaherrera@hotmail.com

ORCID: http://orcid.org/0000-0003-3070-6904

3. Enfermera, Unicesar. Especialista en Cuidado Crítico; magíster en Educación con Enfasis en Cognición, Uninorte. Doctora en Ciencias de la Educación. Docente titular, Universidad Popular del Cesar. Investigadora, grupo Control de Calidad de los Procesos en Salud.

Correo electrónico: mariemmasocarras@unicesar.edu.co

ORCID: http://orcid.org/ 0000-0002-5315-6055 
identificar rasgos diferenciadores para la maestría a ofertar para la salud global. Estas caracteriscticas se identificaron a partir de la investigación holística con enfoque cualicuantitativo, con revisión documental y entrevista. De esta labor emergen elementos que caracterizaron componentes de formación relacionados con dos categorías centrales: cuidado de la vida y sostenibilidad como objeto de estudio.

Palabras clave: problemas sociales, rasgo, educación, sostenibilidad, salud global.

\section{Proposing Features for the Master's Care of Life as a Need for the Context}

\section{Abstract}

It is up to the university - due to social responsibility in a context affected by poor socioeconomic behavior, weak productive and competitive capacity, and social problems that reflect its multifactoriality in health care - to lead the creation of a multidisciplinary master's degree starting with a study of viability that responds to global challenges such as intercultural dialogue, public health, climate change, food security, natural resource management, among others, that promotes the development of critical, creative and innovative thinking with civic, ethical responsibility, and fosters civic culture. As part of this research, training needs and educational trends are analyzed to identify differentiating features for the master's degree to offer for global health. These characteristics were identified from holistic research with a qualitative-quantitative approach, with documentary review and interview. From this work emerged elements that characterized training components related to two central categories: care for life and sustainability as an object of study.

Keywords: social problems, trait, education, sustainability, global health 


\section{Introducción}

Las directivas académico-administrativas de la Facultad de Ciencias de la Salud de la Universidad Popular del Cesar, pensando en mejorar procesos de formación avanzada tanto de sus egresados como de los profesionales del departamento del Cesar, en concordancia con lo establecido por el Ministerio de Educación Nacional con el Decreto 1330 (2019) —que establece la calidad en función de responder a las demandas sociales, culturales y ambientales para transformar y desarrollar las labores formativas, académicas, docentes, científicas, culturales y de extensión- reconocen al grupo de investigación Control de Calidad de los Procesos en Salud la función de presentar una propuesta de maestría con pertinencia al contexto.

Ofertar un programa de formación avanzada en salud acorde a criterios del Ministerio de Educación de Colombia con pertinencia respecto a las necesidades del contexto plantea como objetivo general analizar la viabilidad, en términos de necesidades de formación y tendencias educativas, de un programa que fundamente rasgos diferenciadores de formación para la salud global.

La revisión documental como fuente primaria sobre tendencias educativas y temáticas de mayor relevancia permitió develar categorías como sostenibilidad, calidad de vida, competitividad, cuidado de la vida, ciudadanía, desarrollo humano, que en proceso de reflexividad se relacionaron con el cuidado de la vida; lo cual, coadyuvado por la formación de enfermeras y gestoras de la línea de investigación Control de calidad de los procesos en salud, que adopta como objeto de estudio la formación en salud y cuidado de la salud, permite precisar el cuidado de la vida como objeto de estudio de la maestría a ofertar.

Desde la mirada de la complejidad, cuidar la salud a lo largo del curso de la vida, desde el razonamiento crítico, científico y holístico, y desde una dinámica integrada y evolutiva, es considerado como una forma de ser y estar con otros, que implica complemen- 
tariedad, interculturalidad, humanización, entre otros (Sánchez y Rondón, 2013). Al igual que este, el concepto de calidad de vida tiene una visión subjetiva o perceptiva y un análisis objetivo desde la salud; por ser un concepto multidimensional, no hay criterios únicos para precisarlo; por tanto, el paradigma que subyace en cada argumentación dará validez a sus fundamentos. Se asume como proyecto una formación que favorezca el desarrollo del pensamiento sistémico (Noboa, 2018) global e incluyente como alternativa indispensable para el trabajo en equipo, con una visión transdisciplinar en las alternativas de solución a los problemas sociales, y el conocimiento glocal como sincronización del conocimiento global e inclusor de las diversas alteridades y juicios de los otros en todas las dimensiones de la vida.

Consecuentemente, la segunda fase cualitativa del estudio investiga necesidades de formación avanzada con empresarios y profesionales que laboran en los diferentes sectores de la economía, principalmente en salud. El análisis de las transcripciones de entrevistas obtuvo el sentido integral de conceptos aplicados a categorías emergentes con temáticas de formación necesarias para comprender y atender problemas sociales complejos y la sostenibilidad, y facilitó develar características potenciales del currículo y un modelo pedagógico coherente con el de la universidad.

Se presenta un avance parcial del análisis, y los rasgos diferenciadores que se relacionan con la complejidad de los fenómenos en salud y de la vida y son consecuentes con el estudio de factibilidad (fase en proceso).

\section{Metodología}

El diseño del estudio acoge de Rivadeneira (2013) el modelo investigativo integrador derivado de la investigación holística de Hurtado (2007); es cualitativo y cuantitativo, contextual e interpretativo; las dos primeras fases son cualitativas, y son avance de la investigación de la que resulta el presente artículo. 
- Unidad de análisis: documentos relacionados al tema de investigación y transcripción de entrevistas.

- Unidad de trabajo: documentos virtuales de programas de formación avanzada, declaraciones de organismos que abordan problemáticas sociales, normativa y tendencias de educación y salud; participantes potenciales tanto de la universidad como del sector empresarial local.

- Población y configuración de la muestra (ver tabla 1):

Tabla 1. Configuración de la muestra del estudio

\begin{tabular}{|c|c|c|c|}
\hline \multicolumn{2}{|l|}{ Unidad de trabajo } & Población & Configuración \\
\hline \multirow{2}{*}{ Documento virtual } & Programas de maestría & 20 & 12 \\
\hline & $\begin{array}{l}\text { Organismos internacional y nacional } \\
\text { relacionados con salud y educación }\end{array}$ & 30 & 16 \\
\hline \multirow{3}{*}{$\begin{array}{l}\text { Transcripciones } \\
\text { de entrevistas a } \\
\text { participantes sobre } \\
\text { necesidades de } \\
\text { formación }\end{array}$} & $\begin{array}{l}\text { Personal administrativo de la universidad } \\
\text { (decanos y directores de programas) }\end{array}$ & 28 & 15 \\
\hline & Sector productivo & 128 & 25 \\
\hline & Sector salud & 76 & 51 \\
\hline
\end{tabular}

Fuente: elaboración propia.

- Fases del proceso correspondiente al avance de prefactibilidad:

- Primera fase en que se realizó análisis inductivo de datos de revisión documental de internet, redes académicas y organismos internacional (declaraciones, artículos, páginas web, tendencias educativas) que permitieron identificar características de los programas académicos (denominación, objetivos, modelo curricular, perfiles, evaluaciones). Las fuentes de información primaria fueron: Objetivos de Desarrollo Sostenible; 
Unesco - lesalc (2019), con su Plan de Acción 20182028; Consejo Privado de Competitividad, con sus indicadores de competitividad; Plan Nacional de Desarrollo 2018-2022, que define pactos por la equidad a cumplir; Ministerio de Ciencias (2019), con la Misión de Sabios; Colciencias (2017), con el Proyecto Formación de Capital Humano de Alto Nivel para la Investigación, el Desarrollo Tecnológico y la Innovación; Ministerio de Salud de Colombia (2018) sobre políticas de formación de talento humano en salud; Programa de Gobierno Alcaldía de Valledupar 2020-2023 Seguridad para Todos; Departamento Nacional de Planeación (Visión Cesar-Caribe 2032); temáticas prioritarias de los diferentes organismos que atienden la salud y educación del orden nacional e internacional; políticas de educación y salud, entre otros.

- La segunda fase fue de análisis exploratorio sobre necesidades de formación en el sector productivo, académico-institucional y del sector externo a nivel local.

- Procesamiento de los datos: en las dos primeras fases se utilizó triangulación de datos de documentos y transcripciones de entrevistas; el análisis inductivo buscó su interpretación y comprensión holística, con aportes metodológicos, pedagógicos, conceptuales, sociales y humanos, e influyó en el diseño curricular en correspondencia con el pensamiento sistémico global incluyente — planteado por Noboa (2018) y Luengo (2018) — del maestrante; dada la interdisciplinariedad de los autores de dichas fuentes primarias y del proceso metodológico complementario con la entrevista de los participantes de diferentes profesiones sobre necesidades de formación avanzada, se realizó proceso de análisis Sico, cognitivos de alto nivel (explorar, describir, analizar e interpretar) para contextualizar 
el objeto de estudio pertinente a los estadios de productividad (comparación, explicación, predecir, proponer, modificar o crear).

De esta forma, emerge una propuesta preliminar de formación innovadora en su denominación y currículo que surgió desde la contextualización del problema, el contexto social, el significado dado por los participantes sobre el tema tratado, sin diferenciación de importancias o criterios intersubjetivos que podían generarse en el análisis e interpretación. Esto favoreció comprender el proceso de formación a ofertar dando respuesta al interrogante de qué dominios se necesitan, para qué, y cómo hacerlo desde la visión educativa transformadora, facilitado del análisis de la espiral holística, acogiendo a Rivadeneira (2013), y de la búsqueda de la formación integral e integrada, configurando así la escala integrativa de los objetivos, conceptos de fases y estadios, y la concatenación de los tipos de investigación como momentos de un proceso único.

Esto configuró un proceso secuencial y continuo que permitió caracterizar inicialmente el conocimiento necesario para un modelo curricular con categorías emergentes del proceso analítico transdisciplinar, dada la complejidad de los fenómenos en salud y de la vida como aporte a la sostenibilidad y calidad de vida, facilitando así rasgos para la respectiva denominación.

\section{Resultados}

\section{Formación holística a tono con la problemática social y al desarrollo tecnológico de la región}

En la revisión documental se encontraron principalmente programas cuyo objeto de estudio es satisfacer las necesidades de la institucionalidad o de la disciplina; se han desarrollado preferentemente programas de maestrías centrados en la profesionalización, pero en su gran mayoría los procesos investigativos y de proyección social son aún incipientes y de poco impacto en sus contextos porque los currículos se deben orientar a ampliar la visión intelec- 
tual combinada con la responsabilidad social y la formación para la convivencia pacífica

El desempeño de los futuros egresados y sus principios se basan en el humanismo, en el bien público, la salud como derecho humano y la base para garantizar la realización de otros derechos (Unesco - lesalc, 2019); esto significa que deben responder a las demandas sociales con conocimientos técnicos, eficientes, oportunos y cuya aplicabilidad apunte hacia la superación de los conflictos y situaciones de afectación social para alcanzar el bienestar común (Unesco - lesalc, 2019, p. 17).

Colombia enfrenta grandes retos en materia de competitividad, productividad e innovación del aparato productivo, y empleabilidad para disminuir la pobreza que incide en el desarrollo económico y social. El Ministerio de Educación Nacional (MEN) (2007) ve necesario poner en marcha una estrategia nacional para la gestión del recurso humano, de tal manera que este puede anticiparse y responder efectivamente a las necesidades productivas y sociales del país.

El Plan de Desarrollo del Departamento del Cesar (2020-2023) reconoce que la institucionalidad se opera de forma deficiente e incoherente con las políticas universales establecidas debido a la mirada disciplinar y la formación tradicional, las cuales han sido asumidas en los planes de salud en el país; afirma que el departamento no cuenta con una cultura industrial competitiva y productiva ni, menos, innovadora, y su sistema de producción agropecuario está basado en bienes primarios y desplazados por el sector minero (extracción de carbón e hidrocarburos), comercio y prestación de servicios principalmente Por esto, requiere del Gobierno impulsar políticas, programas y proyectos para que los habitantes dispongan de alternativas que faciliten el acceso a servicios de salud y educación; generar ingresos, con el consecuente bienestar general; consolidar oferta productiva con oportunidades comerciales y con la apuesta económica del territorio, es decir, sostenibilidad con programas que incentiven la producción. 
Dentro de estas políticas y proyectos se debe incluir la formación de profesionales con un pensamiento complejo, global, incluyente, con visión de pertinencia social, para que se apropien del conocimiento en las diversas áreas al servicio de colectivos, contribuyendo así en la mejoría del estado de salud de la población y en su desarrollo sociocultural. La enseñanza universitaria necesita conocer la opinión de los miembros que la constituyen, y especialmente de los maestrantes, con el propósito de utilizar metodologías adecuadas para que el educando pueda lograr las competencias propuestas como meta de aprendizaje (Aparicio y Ostos, 2018).

Diferentes documentos - como el Plan Regional de Competitividad del Cesar (2008); el de Bechara, Hernández, Vergara y Quintero (2014); el del Consejo Privado de Competitividad y Centro de Pensamiento en Estrategias Competitivas (Cepec) (2018) y otros documentos relacionados-, que permitieron visionar indicadores globales de competitividad para que el currículo a ofertarse sea de suma responsabilidad social, representan un insumo técnico valioso para orientar la formación respectiva (Ministerio de Salud de Colombia, 2018, p. 16): una que contribuya en la focalización de políticas públicas, fortaleciendo capacidades competitivas frente a la globalización e internacionalización de las economías, y que dependerá de la idoneidad o competencia de los profesionales, de los modelos de gestión operativa y administrativa (equipos multidisciplinarios de salud), de incentivos, disponibilidad de insumos y tecnologías básicas para la atención en salud.

Dentro del proceso formativo se deben contemplar alianzas estratégicas con sectores productivos y los de educación, salud, ambiente, entre otros, para contribuir en el desarrollo de mejores condiciones competitivas a partir de la actividad investigativa que se centre en los problemas sociales. Por tanto, este debe ser el componente que cualifique mediante herramientas y conocimientos necesarios para visionar los problemas que afectan el cuidado de la vida en su entorno, con sentido crítico-reflexivo y ético; y que de manera consensuada y creativa lidere proyectos colaborativos que propugnen por la transformación o cambios en los com- 
portamientos que repercutan significativamente en el desarrollo humano y social. Esto debe hacerse visible tan pronto como se reconozcan los efectos de la incorporación de la ciencia y la tecnología y de la reflexión elaborada sobre sus fines en los procesos de trabajo, en la producción de la riqueza material y simbólica y en el desarrollo de la organización social; esta es una de las razones que sustenta por qué la maestría a ofertar debe entretejer todos los componentes de formación.

Lo anterior devela un proceso diferenciador para el programa que tiene que ver con buscar respuestas a la cuestión de qué dominios se espera de los maestrantes, para qué y cómo hacer esa formación (figura 1).

Figura 1. Visión documental para el programa a ofertar.

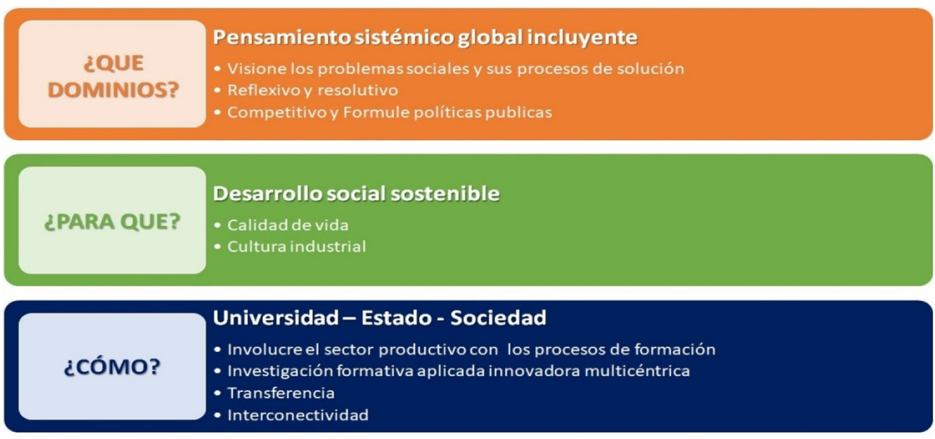

Fuente: elaboración propia a partir del estudio de un programa de maestría a ofertar en la Unicesar (Grupo Control de Calidad de los Procesos en Salud).

El mundo global impone retos para el desarrollo de la ciencia y la tecnología, por lo que es imperante la participación en estudios multicéntricos, y la misión pedagógica es fomentar la generación del nuevo conocimiento, generar incentivos para la creación de nuevos grupos de investigación y nuevos investigadores que contribuyan a transformar factores de riesgo que contaminan la salud, el ambiente y la industria para aportar a la calidad de vida.

Con la segunda fase, el estudio de necesidades de formación, en los participantes se encontró principalmente lo siguiente (tablas 2 y 3$)$. 
Tabla 2. Necesidades de formación expresadas por los participantes como potencial para la maestría a ofertar.

\begin{tabular}{|c|c|c|c|}
\hline \multicolumn{4}{|c|}{ Universidad oferente } \\
\hline \multicolumn{2}{|c|}{ Administrativos (7) } & \multicolumn{2}{|c|}{ Directivos académicos (8) } \\
\hline $\begin{array}{l}\text { Diversificar la oferta } \\
\text { Que no sean } \\
\text { especializaciones }\end{array}$ & $\begin{array}{l}42 \% \\
\text { Agricultura, ganadería, } \\
\text { turismo, acuicultura, } \\
\text { salud, minería, } \\
\text { logística, ambiente, } \\
\text { para responder a } \\
\text { las necesidades de } \\
\text { la región y de sus } \\
\text { características locales, } \\
\text { como paz y el conflicto } \\
\text { que azota tanto a } \\
\text { nuestro departamento } \\
\text { como en el país }\end{array}$ & \multicolumn{2}{|c|}{$\begin{array}{l}\text { Otros programas en salud, ingeniería, área social, minería, } \\
\text { electrónica, de tal manera que integren diversos campos de } \\
\text { conocimiento para una formación integrada y holística. } \\
\text { La universidad goza de buen personal docente tanto en } \\
\text { formación como de experiencia, al igual se cuenta con una } \\
\text { investigación estructurada y de calidad. } \\
\text { La región tiene habitantes con formación avanzada y de } \\
\text { experiencia en las diferentes áreas de desarrollo local, como } \\
\text { es en las áreas agrícola, ambiente y minería; y no seguir } \\
\text { con programas académicos que no están a tono con las } \\
\text { necesidades de la región; además se tiene infraestructura } \\
\text { acorde al desarrollo de formación avanzada. } \\
\text { La competitividad, sostenibilidad son indicadores débiles en } \\
\text { la localidad, requiriéndose de pujanza, cultura emprendedora, } \\
\text { manejo de diferentes idiomas para un buen mercadeo. } \\
\text { Un programa de formación avanzada que se proponga debe } \\
\text { tener temáticas relacionadas a la bioingeniería, comunicación, } \\
\text { redes, telemática, para afrontar el desarrollo de la ciencia y la } \\
\text { tecnología de la localidad. }\end{array}$} \\
\hline \multicolumn{4}{|c|}{ Sector productivo empleador } \\
\hline \multicolumn{3}{|c|}{ Sector salud (6) } & Oros sectores (25) \\
\hline \multicolumn{3}{|c|}{$\begin{array}{l}\text { Desde la institucionalidad se puede estudiar a través de alianzas } \\
\text { estratégicas para contribuir a su solución de los problemas sociales en } \\
\text { cinco áreas críticas: 1) bajo nivel de desarrollo y calidad de vida que se } \\
\text { evidencia de la ciudad y municipios aledaños, que entre más distantes a } \\
\text { Valledupar son más afectados; 2) problemas de contaminación ambiental; } \\
\text { 3) influencia negativa de modos de vida a nivel de habitantes transitorios o } \\
\text { inmigrantes que afectan la calidad del ambiente y de sus recursos naturales, } \\
\text { adoptando estilos de vida cotidiana que no eran parte de sus nativos, entre } \\
\text { otras circunstancias; 4) la ciudad no está preparada para ofrecer calidad } \\
\text { de vida óptima, no hay infraestructura urbanística, ni fuentes de empleo o } \\
\text { generación de empresa porque el nivel de competitividad de lo existente no } \\
\text { alcanza a ofrecer la calidad necesaria en la atención de las necesidades } \\
\text { de toda una comunidad; 5) cultura insostenible en el manejo del ambiente y } \\
\text { recursos naturales. } \\
\text { Consideran que se debe ofrecer programas de biotecnología, agropecuaria, } \\
\text { inmunohematología, calidad analítica en laboratorios, instrumentación } \\
\text { quirúrgica especializada, salud pública, entre otros; que se hagan buenos } \\
\text { convenios entre las demás instituciones. Estos aspectos resultan relevantes } \\
\text { al ser coincidentes con la apreciación de los directivos académicos y } \\
\text { administrativos. }\end{array}$} & $\begin{array}{l}\text { "Necesidad de crear programas de } \\
\text { formación con impacto social, porque } \\
\text { hay una marcada desigualdad } \\
\text { en todas las dimensiones de } \\
\text { proyección humana, que con dicha } \\
\text { formación posibilitaría la diversidad } \\
\text { de cambios necesarios, facilitado } \\
\text { por la investigación; la visión } \\
\text { interdisciplinar e interprofesional } \\
\text { ayudaría a forjar profesionales con } \\
\text { mayor sensibilidad, conscientes } \\
\text { de su rol como persona, habitante, } \\
\text { y profesional en su proyección y } \\
\text { realmente ser forjadores de un } \\
\text { mundo sostenible que sea como de } \\
\text { ciudadano formador de generaciones } \\
\text { y de convivencia cotidiana en el } \\
\text { desarrollo de la sociedad". }\end{array}$ \\
\hline \multicolumn{4}{|c|}{$\begin{array}{l}\text { Interpretación: justifican ofrecer un programa de formación avanzada que aborde el conocimiento integrador } \\
\text { para la solución de las necesidades que más afectan a la sociedad de hoy tanto local, regional como nacional, } \\
\text { porque el fondo de su problemática radica en las prácticas de proyección humana que destruyes la naturaleza y } \\
\text { no hay convivencia pacífica ni manejo de la ética social necesaria para una economía sostenible, competitiva y de } \\
\text { convivencia pacífica, proactiva y emprendedora. Es imperativo un programa de formación avanzada que apunte a } \\
\text { la solución de problemas socioambientales y de desarrollo tecnológico, producto de investigación e innovación que } \\
\text { sea pertinente a las necesidades locales. } \\
\text { El programa de formación avanzada a ofrecer debe cubrir necesidades de las áreas agropecuaria, ganadería, } \\
\text { turismo, minería, salud, ambiente, logística y de paz y conflicto de manera integrada, que facilite una proyección } \\
\text { profesional diversa y en diferentes campos de acción tradicional porque cuidar la vida y la salud no es solo del } \\
\text { sector salud sino de todos. }\end{array}$} \\
\hline
\end{tabular}

Fuente: elaboración propia, resultado de revisión bibliográfica y entrevistas realizadas por el grupo investigador. 
Tabla 3. Motivaciones de personal del sector salud para continuar con una formación avanzada.

¿Qué les motiva continuar con una formación avanzada y sea ofrecida por la Universidad Popular del Cesar?

Participantes de las 3 empresas públicas y 3 privadas

Sí: 42; No: 3

Respuestas Sí:

- Integración de conocimiento en los procesos del cuidado (3)

- Su institución aspira ser hospital universitario (2)

- Porque es abierta a todos los profesionales (5)

- Porque prestaría una mejor función como médico (3)

- Porque a través de la investigación se pueden identificar y resolver más problemas (3)

- Permite crecimiento personal, institucional a través de mejorar la calidad de vida (3)

- Muy buena por la interdisciplinariedad que lleva a mejorar los problemas sociales desde diferentes ámbitos (3)

- Porque a pesar de mi función de atender público, manejo los problemas sociales desde diferentes áreas de campo, porque mis funciones profesionales se articulan con el desarrollo humano, la calidad y el enfoque cultural que tiene la población indígena (5)

- Porque deseo que mi profesión se contextualice (2)

- Interesante porque se involucra al individuo y a la sociedad (2)

- Porque la sostenibilidad es el desarrollo que nos invita a satisfacer necesidades del presente sin comprometer las capacidades de las futuras generaciones (2)

- Porque las actividades y funciones que desempeñan en el ente territorial a través de la maestría podrían visionar las necesidades apremiantes de la comunidad para que se logre un nivel de vida justo (3)

- Porque el desarrollo de toda empresa, familia, persona es necesario para ayudar en la sostenibilidad (2)

- Porque se les permite sus cambios de turnos apoyándoles en el desarrollo de la maestría (2)

- Mejora su acción profesional, laboral y cualifica los procesos con vida saludable, favoreciendo calidad de vida (2)

Respuesta No:

- Porque tiene otras aspiraciones

- Su interés es maestría sobre Finanzas Públicas

- Falta de capacitación

Interpretación: existe motivación desde la proyección profesional que redunde en la calidad de la atención humanizada y de trabajo en equipo interprofesional y como empresa.

Existe conciencia de la calidad que debe impartirse en la proyección profesional y disciplinar y desde la empresa, porque implícitamente ven la necesidad de mejorar la calidad de vida, de la proyección de los usuarios y de la empresa en un ambiente de corresponsabilidad y de sus condiciones en función de la sostenibilidad, pero es necesario fortalecer la motivación para su crecimiento personal, de rigor ético, inclusor y humano, porque se está traspasando la rentabilidad y competitividad desde lo profesional y empresarial, mas no como ciudadano que multiplica su ejemplo de vida con la sociedad.

Fuente: elaboración propia a partir de entrevistas realizadas por el grupo investigador. 
La universalización del conocimiento, la equidad y la justicia pueden ser resueltas con la inclusión de las TIC, que puestas a disposición de la comunidad educativa motivarían la innovación, así como reinterpretar y resignificar datos conseguidos en su investigación (Ferreyra y Besso, 2014) y favorecer la inteligencia interactiva.

\section{Formación en investigación con responsabilidad social: innovación y transferencia}

Los investigadores Torres y Sánchez (2014) afirman que la responsabilidad social universitaria, asumida como política integral de la universidad, desarrolla conciencia acerca del papel que nos atañe como miembros de una comunidad académica que hoy día navega en el inmenso mar de la incertidumbre impuesta por los avances tecnológicos y la revolución informática en un mundo global, con grandes desafíos por ser el motor de desarrollo social, y por ende del proceso de formación al que se le impone renovación, a veces sin la mejor orientación.

El proceso de formación se dirige a dotar al maestrante con competencias investigativas que lo conduzcan a analizar, interpretar, comprender y reflexionar la problemática social detectada, con el propósito de definir estrategias pedagógicas, asumir enfoques de investigación y teorías que lo empoderan para gestionar su conocimiento; conformar e interactuar con un equipo de trabajo interprofesional o intersectorial ante el cual puede argumentar su propuesta innovadora y establecer negociación a través de acuerdos de alianza estratégica; aportar saberes para solucionar los problemas detectados en una visión de sostenibilidad de la calidad de vida en congruencia con la confluencia de las funciones misionales de educación superior. Esto impone la necesidad de transformar tanto los enfoques curriculares como la práctica misma de los estamentos universitarios y el enfoque de la universidad, para que la práctica de docentes, estudiantes y administrativos sea correspondiente con las necesidades sociales (Herrera, Sánchez y Socarrás, 2017). 
A pesar que en el nivel de desarrollo de la ciencia y la tecnología el departamento del Cesar es muy deficiente para responder a sus propias necesidades y muy distante al desarrollo de otras ciudades, esta es una condición para que se le reconozca a la universidad su gran aporte al desarrollo social de la región, y ofrezca un programa de formación avanzada que haga gran aporte a la transformación que se necesita para el cuidado de la vida, del ambiente, de los recursos naturales, dada su infraestructura, tecnología y recurso humano. Por lo anterior, emergen algunas características diferenciadoras potenciales para el programa de formación avanzada a ofertar (figura 2).

Figura 2. Formación en investigación y componentes para la maestría propuesta.

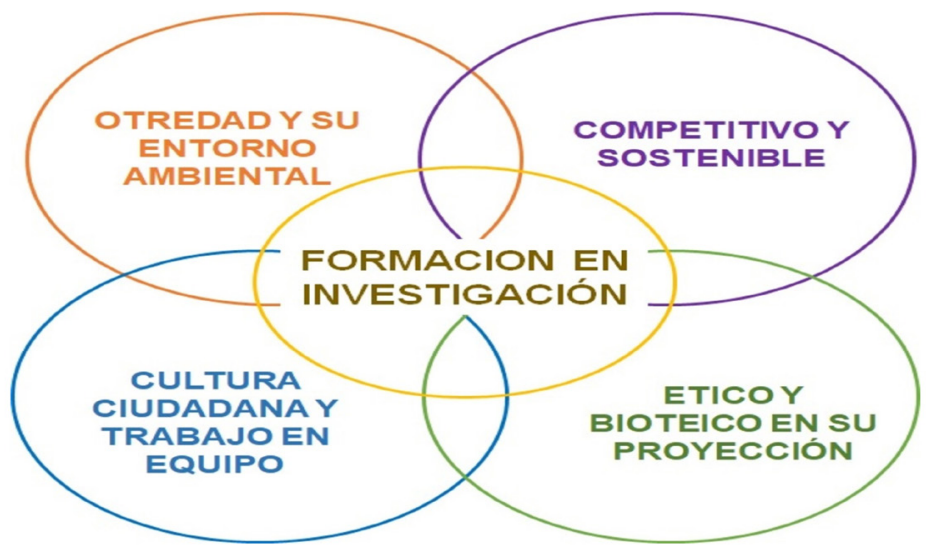

Fuente: elaboración propia a partir del estudio de un programa de maestría a ofertar en la Unicesar (Grupo Control de Calidad de los Procesos en Salud).

Se parte de la ética y responsabilidad social, repensando el compromiso con la institución, pero el objeto de estudio a elegir para la formación, en tanto los fenómenos de la salud son complejos y están imbricados en el cuidado, también exige entre otros aspectos claridad conceptual y precisión.

Con el legado de los avances en formación avanzada y experticia, los rasgos propuestos en términos de pedagogía, roles, perfiles, problemática social, responsabilidad social universitaria, calidad de vida, objetivos del desarrollo sostenible y la mirada ho- 
lística para sus abordajes, y la bioética como reflexión sobre el accionar personal cotidiano para proteger la vida, son aspectos que permitieron precisar que el objeto de estudio fuese el cuidado de la vida, pensando en formar ciudadanos globales con perfiles de desempeño de mayor complejidad a la disciplinar; se decide que el desarrollo social sostenible sea una meta para la proyección del futuro egresado favorecido con el aprendizaje solidario y el fomento del pensamiento sistémico global incluyente, que le da un rasgo diferenciador para un desempeño de mayor amplitud y complejidad.

\section{Formación de maestría requiere de una visión complementaria y diferenciadora}

La investigación documental sobre programas en Colombia en el área de la salud que responden a las necesidades del sistema de salud y educación, y reconocen la formación integral desde lo disciplinar o para subáreas de atención especializada, no son relevantes para la transformación de los perfiles de egresados que se espera.

El desarrollo de pedagogías que cubran la dimensión personal, no solo lo valorativo, con la capacidad crítica, creativa, emprendedora e innovadora; la interpersonal para el trabajo en equipo y la inclusión; y la dimensión ecológica para desarrollar conciencia social visible, con su propuesta de formación investigativa y de emprendimiento para una proyección interprofesional con abordajes holísticos a problemas sociales y de afectación al entorno, acoge propuestas y sugerencias de organismos encargados de la ciencia, tecnología e innovación, y de emprendimiento de corte nacional, a saber:

- El Ministerio de Ciencias (antes Colciencias) (2017) en Colombia propone intervenir disminuyendo el rezago que existe en ciencia, tecnología e innovación en el país por medio de focalizar la creación y 
fortalecimiento de capacidades tanto en capital humano como infraestructura, financiación y cultura de la CTel. Espera, en consecuencia, que entre el 2018 y el 2022 se hayan mejorado capacidades existentes y se multipliquen los impactos en investigación e innovación: creando redes de ciencia, tecnología e innovación; clúster tecnológico en áreas focalizadas y ciudades con vocación marcada hacia la generación permanente de conocimiento e innovación; empresas y tecnologías como estrategias competitivas a largo plazo.

- El Consejo Privado de Competitividad y el Centro de Pensamiento en Estrategias Competitivas (Cepec) de la Universidad del Rosario (2018) reconocen que algunas ciudades del Caribe como Montería, Riohacha, Santa Marta, Sincelejo y Valledupar están clasificadas en la etapa 1, con menor desarrollo en cuanto a competitividad.

- El Plan de Acción de la Comisión de Regulación de la Educación Superior (Unesco - Iesalc, 2019) plantea la necesidad de hacer cambios curriculares apropiados para mejorar la calidad educativa de América Latina y el Caribe.

- El documento Misión de Sabios (Ministerio de Ciencias, 2019) le plantea a la universidad local cambiar paradigmas partiendo de la noción de que se quiere construir como nación desde el trabajo local y reflexionar sobre su responsabilidad social en la calidad de vida regional y con su aporte cultural, argumentos que convalidan esta concepción de proponer un currículo innovador con estructuras académicas y administrativas confluyentes en la transformación para gestar una práctica profesional renovada, buscando impactos a mediano y largo plazo sobre el desarrollo humano y social. 
- El Plan Nacional de Desarrollo (2018-2022) describe veintinueve pactos para el desarrollo de Colombia referenciados y acogidos para la formación del maestrante; muy relacionados con los componentes y núcleos de formación, correspondientes a desarrollo social sostenible, calidad de vida, ética para la convivencia paz y justicia, cultura ciudadana, competitividad e innovación, humanización de los procesos de atención, inclusión y género, y valoración de la otredad en la construcción de ciudadanía.

El compromiso pleno con la responsabilidad social supone asumir que la universidad no es un ente independiente, sino una parte más de la sociedad que debe responderle a esta y a la comunidad; sugiere ofrecer formación de profesionales con visión holística que asuman la tarea de reconstruir el tejido social (Giuffre y Ratto, 2014) y aborden la complejidad de los fenómenos en salud y de la vida para lograr un mundo sostenible y justo que aclama la existencia de lo humano y no humano como un todo íntegro, equilibrado y razonable; y a su vez, repensar un currículo transformador con modelo pedagógico ajustado al institucional (cognitivo-contextual de corte constructivista).

Sánchez et.al. (2015) manifiestan que abordar la responsabilidad social de la Facultad de Salud como estamento universitario supone asumir una postura global frente al entorno, que lleva a cada uno de sus actores a adentrarse en su organización para comprender las diversas misiones que les atañe en función de una sociedad altamente saludable y empoderada para vivir más y vivir mejor. Además, obliga a la academia a repensar cómo es su cultura organizacional, con qué políticas de carácter social, medioambiental, laboral y económicas se cuenta para aportar a la necesidad de transformar la realidad que agobia día a día generando en la colectividad desesperanza y falta de credibilidad institucional; y requiere una visión integral del desempeño pertinente y ético, entrelazar las funciones misionales de docencia, investigación, extensión y conocer la opinión de los estudiantes para lograr cambios en las metodologías de enseñanza-aprendizaje e innovaciones docentes, 
así como favorecer el aprendizaje significativo en los estudiantes (Valdivieso, Montoya y Martínez, 2018).

Se requiere exaltar en el objeto de estudio la clara conciencia con que se deben orientan las acciones, elecciones y decisiones de hombres y mujeres como ciudadanos globales, integrando su territorio como el entorno natural, posibilitando la vida, la integridad y la salud como premisas fundamentales para el desarrollo humano en lo individual y colectivo; y adicionalmente considerar la responsabilidad social universitaria (Naval y Ruiz, 2013) como una política de gestión ética integral y transversal de las tres funciones sustantivas y de la administración central de la universidad que implique un mayor compromiso con la sociedad y con la contribución a un nuevo modelo de desarrollo más equilibrado y sostenible (p. 16).

La Universidad Popular del Cesar posee un gran potencial en investigación y docencia, que desde la interdisciplinariedad y el fomento del trabajo en equipo puede potenciar el objeto de estudio de la maestría con investigación basada en la evidencia para ser más pertinente en la búsqueda de mejor asertividad y de alternativas de solución y emprendimiento de transformación, evidenciando el cuidado de la vida en las comunidades a través del aprendizaje y el servicio (Torres-Rivera, 2015), con nuevos enfoques curricular e investigativo, así como con la proyección hacia sectores productivos, de servicios y a la sociedad. Por tanto, hay potencial para determinar una política institucional de gestión sobre responsabilidad social universitaria (Medina-Peña et al., 2017).

El cuidado de sí y el cuidado de los otros es un valor de ciudadanía; atañe a cada disciplina o profesión asumirlo desde lo público como un derecho, repensar el cuidado desde lo político, y aprovechar que las áreas de conocimiento del desarrollo humano se formulan desde la filosofía, como el buen vivir que conduce a la felicidad. La ciudadanía, que en un estado de bienestar acoge normas y valores para buscar la convivencia pacífica de igualdad y justicia, y la política, como calidad de vida para el desarrollo, constituyen la mirada integral del ser humano (Franco-Peláez, 2009) 
como totalidad imposible de fragmentar debido a su dignidad e identidad cultural.

El desarrollo local requiere fomentar innovación, capacidad emprendedora, calidad del capital humano y flexibilidad del sistema productivo (Barragán y Ayaviri, 2017), que respondan a los cambios continuos que la tecnología ocasiona en el ambiente, economía y sociedad, triángulo objeto de la sostenibilidad; y hacerlo con postura filosófica que guíe el abordaje del análisis bioético del desarrollo sostenible, rescate la importancia de la especie humana sin desconocer el deber moral por las diversas formas de vida y de preservación en condiciones de bienestar (Estrada-Cely, SánchezCastillo y Gómez-Cano, 2019), e impulse proyectos de dimensión pertinente para transformar el sistema económico local. Para el logro de estas acciones la universidad posee un gran potencial de investigadores y semilleros que pueden ser focalizados para la inclusión en la maestría en estudio de referencia.

\section{Maestría a ofertarse en el modelo educativo holístico transformador}

Se planteó la necesidad de asumir la formación transformadora basada en el modelo holístico de gestión pedagógica constructivista (Gluyas-Fitch, Esparza-Parga, Romero-Sánchez y Rubio-Barrios, 2015) y en el desarrollo del pensamiento sistémico global incluyente, con visión de pertinencia social para apropiar el conocimiento en las diversas áreas al servicio de los colectivos, contribuyendo en la mejoría del estado de salud y en su desarrollo sociocultural, requiriendo desarrollar la meta de reflexión guiada por el mundo real en términos de totalidades para su análisis, comprensión y accionar.

Los problemas globales cada día se tornan más complejos y están mayormente interconectados, como sucede con los de tipo ambiental (cambio climático, demanda de energía, crisis del agua, crecimiento poblacional, entre otros). La sectorización, las fronte- 
ras geográficas en contravía a la misma globalización, el etnocentrismo y antropocentrismo, los tipos de ocupación y trabajo, las presiones de competitividad y rentabilidad por encima del bienestar humano y la calidad de vida dificultan el logro integral de los objetivos de desarrollo sostenible; y son obstáculos para alcanzar metas más integrales y el enfoque sistémico necesario para el buen vivir, que cobije la dimensión humana, ética, y holística del relacionamiento de los seres humanos con su naturaleza, en una convivencia transformada y para siempre (Dávalos, 2011; Cubillo, Hidalgo y Domínguez, 2014).

El tradicional modelo de formación avanzada presenta necesidad de cambio hacia uno que forme con y para la interculturalidad, el trabajo en equipo, cultura ciudadana, ética, corresponsabilidad, conciencia social, otredad, convivencia pacífica, la bioética, el buen trato con los demás componentes de la naturaleza y las prácticas formativas solidarias para el fomento de la conciencia social.

Los documentos de referencia descritos han sido los mayormente decisivos en ese proceso de análisis inductivo para reconocer el eje principal u objeto de estudio cuidado de la vida, que triangulado con la percepción y visión de sus docentes y siendo consecuentes con la responsabilidad social universitaria en la misión de investigación, docencia y proyección social, así como de su coherencia intermisional, permitieron precisar categorías emergentes que entrelazaran la investigación formativa en todo el currículo con rasgos conceptuales precisos que fueran parte de los componentes de formación de la maestría en estudio, como sostenibilidad, cultura ciudadana, ética, innovación y desarrollo, competitividad e interprofesionalización (figura 3). 
Figura 3. Formación en investigación transversal a los componentes formativos de la maestría a ofertarse en la Unicesar.

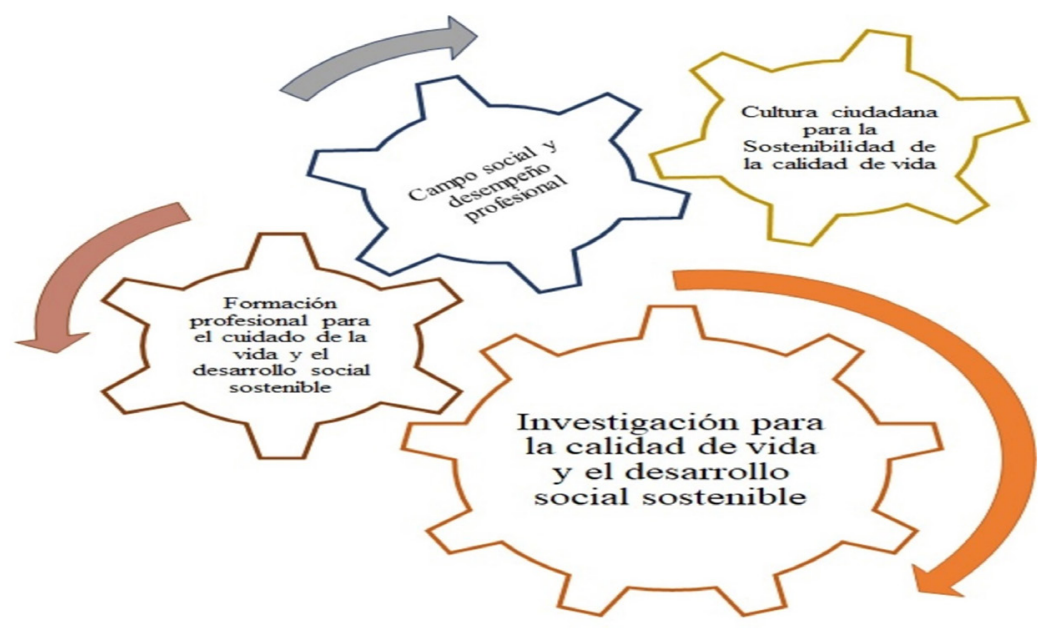

Fuente: elaboración propia a partir del estudio de un programa de maestría a ofertar (Grupo Control de Calidad de los Procesos en Salud).

Este enfoque está relacionado con la complejidad como método de pensamiento (que consiste básicamente en el aprendizaje del pensamiento relacional) y la complejidad como cosmovisión (consideraciones holistas emergentes del pensamiento sistémico), puesto que a criterio de los autores se requiere de una educación holística transformadora que genere conciencia social y responsabilidad para la adopción de un pensamiento sistémico global incluyente.

Posteriormente, en ese proceso de análisis inductivo se propone un currículo transformador y holístico que concebido desde la reflexividad, genere conciencia y responsabilidad con la adopción de un pensamiento sistémico, global e incluyente, mediante el modelo pedagógico institucional (cognitivo-contextual de corte constructivista); y que con estrategias metodológicas activas para el aprendizaje significativo, colaborativo y solidario fomente en el maestrante la tan necesaria conciencia social y el pensamiento sistémico global referido (figura 4) 
Figura 4. La educación holística y transformadora, clave para generar conciencia y responsabilidad para la adopción de un pensamiento sistémico global e incluyente.

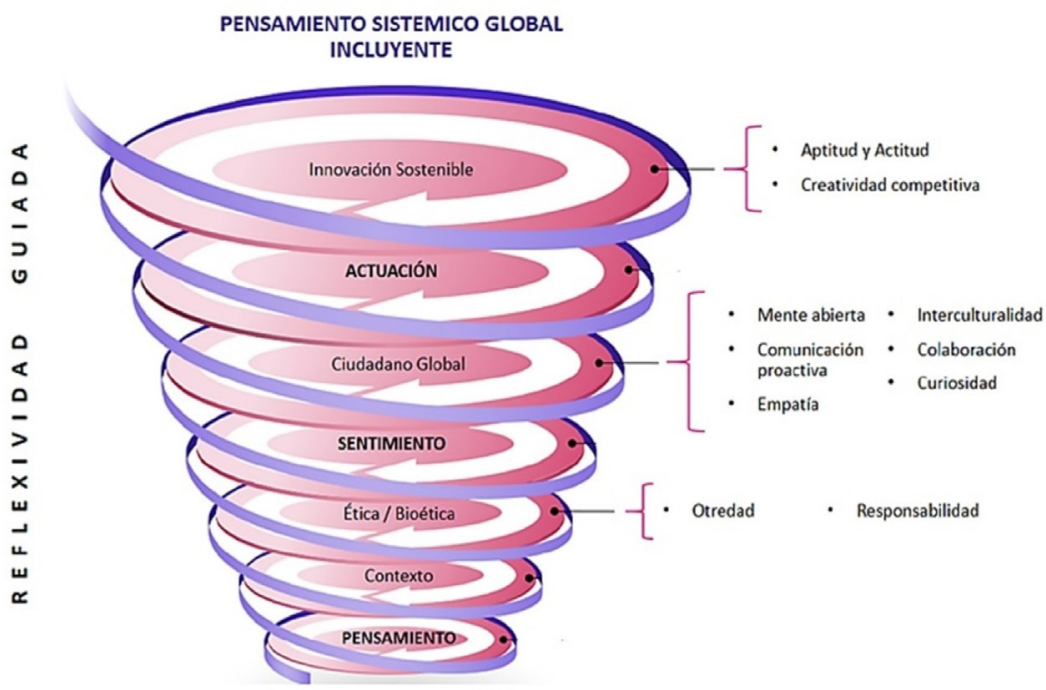

Fuente: elaboración propia a partir del estudio de un programa de maestría a ofertar (Grupo Control de Calidad de los Procesos en Salud).

El programa de formación que emergió de triangular la información entre la primera y la segunda fase del proceso metodológico ya cuenta con la identificación de sus rasgos diferenciadores, además de asumir el cuidado de la vida como objeto de estudio, y tiene una perfecta conexión ideológica, estratégica y práctica con el desarrollo social sostenible (ONU, 2019) y descansa en el modelo educativo institucional, es decir, el cognitivo-contextual de corte constructivista.

El cuidado de la vida y la salud es un fenómeno complejo porque entran en juego múltiples dimensiones relacionadas con la cultu$\mathrm{ra}$, el mundo subjetivo de las personas, las condiciones de vida, la calidad de vida, y demás factores que conforman el desarrollo económico; es decir, depende de las personas y de los contextos desde donde se conceptualiza y acontece en el denso tejido social y ecológico en el que transcurre la historia personal. 


\section{Conclusión}

Desde el principio de la responsabilidad social se enfrentan intersectorialmente grandes desafíos relacionados con la competitividad, productividad e innovación, especialmente en los sistemas productivos y laborales, razón por la cual crear una maestría que vincule al sector educativo, empresarios, Gobierno y sociedad en general es vital para mejorar las relaciones entre universidad, Estado, empresa y sociedad y así impulsar de forma sostenida el desarrollo económico y social de la región, y por ende del país.

En el contexto del departamento del Cesar, el desarrollo de la ciencia y la tecnología es incipiente y no se sabe aprovechar las ventajas competitivas; un programa de formación de maestría en investigación aplicada y con responsabilidad social, además de producir impactos en corto tiempo en la región por ser el cuidado de la vida su objeto de estudio, producirá un valor agregado en la productividad, en la calidad de vida de la población, en el desarrollo social sostenible aunado a la innovación y transferencia del conocimiento

Entre los rasgos diferenciadores potenciales está que la formación debe ser holística, pensando en formar ciudadanos globales con perfiles de desempeño de mayor complejidad a la disciplinar, a tono con la problemática social y avances del desarrollo tecnológico de la región. La maestría a ofertarse requiere ser de una visión complementaria y diferenciadora, con un modelo educativo holístico transformador para que sus maestrantes desarrollen el pensamiento sistémico global e incluyente.

\section{Referencias bibliográficas}

Aparicio, O. Y. y Ostos, O. (2018). Las TIC como herramientas cognitivas para la investigación. RIIEP: Revista Interamericana de Investigación, Educación y Pedagogía, 11(1). DOI: https://doi.org/10.15332/s1657107X.2018.0001.08 
Asamblea Departamental del Cesar. (21 de marzo de 2020). Ordenanza 208: Plan de Desarrollo Departamental del Cesar 2020-2023 "Lo Hacemos Mejor". Recuperado de https://cesar.gov.co/d/filesmain/plan_ desarrollo/ordenanza_208_21_mar_2020.pdf

Barragán, M. y Ayaviri, V. (2017). Innovación y emprendimiento, y su relación con el desarrollo local del pueblo de Salinas de Guaranda, Provincia Bolívar, Ecuador. Información Tecnológica, 28(6), 71-80. DOI: https://dx.doi.org/10.4067/S0718-07642017000600009

Bechara, B., Hernández, A., Vergara, V. y Quintero, J. (2014). Indicador global de competitividad de las ciudades del Caribe colombiano: evolución 2009-2010. Cartagena: Observatorio del Caribe Colombiano; Cámara del Comercio de Cartagena.

Colciencias (Departamento Administrativo de Ciencia, Tecnología e Innovación). (2017). Proyecto Oferta Colciencias: Formación de Capital Humano de Alto Nivel (Doctorado y/o Maestría Investigativa) para la Investigación, el Desarrollo Tecnológico y la Innovación.

Consejo Privado de Competitividad y Centro de Pensamiento en Estrategias Competitivas (Cepec). (2018). Índice de competitividad de ciudades. Bogotá: Universidad del Rosario.

Cubillo, A. P., Hidalgo, A. L. y Domínguez, J. A. (2014). El pensamiento sobre el Buen Vivir: entre el indigenismo, el socialismo y el posdesarrollismo. Revista del CLAD Reforma y Democracia, 60, 27-58.

Dávalos, (2011). Sumak kawsay (la vida en plenitud). En S. Álvarez (ed.), Convivir para perdurar: conflictos ecosociales y sabidurías ecológicas (pp. 201-214). Barcelona: Icaria.

Departamento Nacional de Planeación (DNP) (2008). Plan Regional de Competitividad en el Departamento del Cesar. Convenio de Cooperación 032.

Departamento Nacional de Planeación (DNP) (2019). Plan Nacional de Desarrollo 2018-2022: pacto por Colombia, pacto por la equidad. 
Recuperado de https://www.dnp.gov.co/DNPN/Paginas/Bases-del-PlanNacional-de-Desarrollo.aspx

Estrada-Cely, G. E., Sánchez-Castillo, V. y Gómez-Cano, C. A. (2019). Bioética y desarrollo sostenible: entre el biocentrismo y el antropocentrismo y su sesgo economicista. Clío América, 12(24), 255267. DOI: https://doi.org/10.21676/23897848.2999

Ferreyra, H. A. y Besso, M. (2014). Mesas socioeducativas para la inclusión y la igualdad: un programa "De todos con todos", una experiencia en construcción. RIIEP: Revista Interamericana de Investigación, Educación y Pedagogía, 7(2). DOI: https://doi.org/10.15332/s1657107X.2014.0002.01

Franco-Peláez, R. Z. (2009). La bioética: ética del cuidado de la vida y la salud para el desarrollo humano. Revista Hacia la Promoción de la Salud, 14(2), 75-92.

Giuffre, L. y Ratto, S. (2014). A new paradigm in higher education: University social responsibility (USR). Journal of Education and Human Development, 3(1), 231-238.

Gluyas-Fitch, R. I., Esparza-Parga, R., Romero-Sánchez, M. C. y RubioBarrios, J. E. (2015). Modelo de educación holística: una propuesta para la formación del ser humano. Revista Actualidades Investigativas en Educación, 15(3), 1-25.

Herrera, F., Sánchez, M. y Socarrás, M. (2017). El valor de la responsabilidad social universitaria en la formación de enfermería. Ponencia presentada en la XIV Conferencia Iberoamericana de Educación en Enfermería, Lima, Perú.

Hurtado, J. (2007). Metodología de la investigación: una comprensión holística. Caracas: Quirón; Fundación Sypal.

Luengo, E. (2018). Las vertientes de la complejidad. Guadalajara: Universidad Jesuita de Guadalajara - ITESO. 
Medina-Peña, R., Franco-Gómez, M., Torres-Barreiro, L., VelázquezRodríguez, K., Valencia-Vera, M. y Valencia-Vera, A. (2017). La responsabilidad social universitaria en la actual sociedad del conocimiento: un acercamiento necesario. MediSur, 15(6), 786-791.

Ministerio de Educación Nacional (2007). La revolución educativa: visión 2019. Recuperado de https://www.cepal.org/socinfo/noticias/ noticias/3/32383/Colombia.pdf

Ministerio de Salud de Colombia (2018). Política Nacional de Talento Humano en Salud. Recuperado de https://www.minsalud.gov.co/ sites/rid/Lists/BibliotecaDigital/RIDE/VS/TH/politica-nacional-talentohumano-salud.pdf

Ministerio de Ciencias (2019). I Cumbre Misión Internacional de Sabios. Recuperado de https://minciencias.gov.co/mision_sabios/semana_ sabios

Naval, C. y Ruiz Corbella, M. (2013). La responsabilidad social universitaria, vía para el cuidado de la comunidad. En J. A. Ibáñez (ed.), Educación, libertad y cuidado. Madrid: Dykinson.

Noboa, A. (2018). Pensamiento sistémico, complejidad y ciencias sociales: las bases epistemológicas de las metodologías participativas. De Prácticas y Discursos: Cuadernos de Ciencias Sociales, 7(9). DOI: http://dx.doi.org/10.30972/dpd.792799

ONU (Organización de las Naciones Unidas( (2019).

Rivadeneira, E. M. (2013). Modelo investigativo integrador derivado de la investigación holística. Revista Negotium, 9(26), 116-142.

Sánchez, M. y Rondón, B. (2013). La diversidad cultural en los procesos de formación académica de enfermería requiere el manejo de la ética pedagógica, la corresponsabilidad y un pensamiento mediador. Enfermería Global, 12(29), 147-157. 
Sánchez, M., Hernández, S. N., Galindo, O. J., Romero, D. A., Lozano, G. L., Vence, D. N. et.al. (2015). Responsabilidad social universitaria para el cuidado de la salud de los habitantes área urbana de Valledupar. Valledupar: Unicesar.

Torres, A. G. y Sánchez, L. M. (2014). La responsabilidad social universitaria desde su fundamentación teórica. Libre Empresa, 11(1), 69-105

Torres-Rivera, M. S. (2015). Las voces de los decanos en torno al uso del aprendizaje en servicio para el logro de la responsabilidad social como tercera misión de la universidad (tesis de doctorado). Universidad del Turabo, Gurabo, Puerto Rico.

Unesco - lesalc (Instituto Internacional de la Unesco para la Educación Superior de América Latina y el Caribe). (2019). Plan de Acción CRES 2018-2028. Recuperado de https://www.iesalc.unesco.org/2019/02/22/ plan-de-accion-cres-2018-2028/

Valdivieso, M., Montoya, C. y Martínez, P. (2018). Estudio de percepción de las metodologías de enseñanza-aprendizaje en un postgrado de ingeniería. Revista Interamericana de Investigación, Educación y Pedagogía, 12(2). DOI: https://doi.org/10.15332/25005421.5016

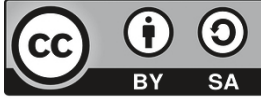

